\title{
Non-Local Scattering Kernel and the Hydrodynamic Limit
}

\author{
Maria Carmela Lombardo • Russel E. Caflisch • \\ Marco Sammartino
}

Received: 6 January 2007 / Accepted: 7 June 2007 / Published online: 3 October 2007

(C) Springer Science+Business Media, LLC 2007

\begin{abstract}
In this paper we study the interaction of a fluid with a wall in the framework of the kinetic theory. We consider the possibility that the fluid molecules can penetrate the wall to be reflected by the inner layers of the wall. This results in a scattering kernel which is a non-local generalization of the classical Maxwell scattering kernel. The proposed scattering kernel satisfies a global mass conservation law and a generalized reciprocity relation. We study the hydrodynamic limit performing a Knudsen layer analysis, and derive a new class of (weakly) nonlocal boundary conditions to be imposed to the Navier-Stokes equations.
\end{abstract}

Keywords Boltzmann equation · Hydrodynamic limit · Navier-Stokes flow · Nonlocal boundary conditions

\section{Introduction}

The motivation for this paper comes from the fact that the problem of finding the appropriate boundary conditions for the Navier-Stokes equations is still an active area of research and poses many interesting questions.

In fact, despite of their overwhelming success in most practical cases, the no-slip boundary conditions usually imposed for the Navier-Stokes Equations (NSE), fail to correctly describe the complicated interactions between the fluid and a solid boundary in many different situations like geophysical models,turbulence modeling and micro and nano-fluidics.

The aim of this paper is to derive non-local boundary conditions for the Navier-Stokes equations starting from a kinetic description based on the Boltzmann equation. The relation

M.C. Lombardo $(\bowtie) \cdot$ M. Sammartino

Dipartimento di Matematica, Università di Palermo, Via Archirafi 34, 90123 Palermo, Italy

e-mail: lombardo@math.unipa.it

M. Sammartino

e-mail: marco@math.unipa.it

R.E. Caflisch

Department of Mathematics, UCLA, 520 Portola Plaza, Los Angeles, CA 90095, USA

e-mail: caflisch@math.ucla.edu 
of NSE with the Boltzmann equation has been discussed by many authors (see [5, 22] and references therein): it is well known that the NSE can be obtained performing an asymptotic expansion of the Boltzmann equation in the limit as the Knudsen number tends to zero. The different boundary conditions (no-slip, free-slip, Robin) are obtained from the properties of the solution of the Boltzmann half space problem inside the Knudsen layer (the theory of the asymptotic limit of the boundary-value problem for a steady gas is treated, for example, in $[11,16,20,21])$.

Starting from the Boltzmann equation we shall derive Robin-type boundary conditions for the macroscopic variables which take into account the effect of nonlocal interactions at the wall. We shall investigate the steady behavior of the fluid on the basis of the Boltzmann equation on a 3-dimensional half space. We shall introduce a simple model for the interaction of the fluid-boundary interaction whose corresponding scattering kernel generalizes the classical Maxwell's interaction law. The proposed kernel is shown to satisfy a nonlocal mass conservation and a generalized reciprocity relation. We shall also perform an asymptotic expansion in the Knudsen number leading, in the hydrodynamical limit, to new boundary conditions for the macroscopic variables.

The core of this paper consists of Sect. 4 and Sect. 5 where we propose a model for the interaction of fluid particles with a wall which, we believe, can be of interest in micro and nano-fluidics. The problem of the derivation of the appropriate boundary conditions in micro-fluidics or nano-fluidics has recently attracted a lot of interest both from the experimental (see e.g. the review in [13]) and the theoretical point of view. Molecular dynamics (MD) has become an important tool for investigating the behavior of a fluid at the boundary. In the MD setting the wall is considered as a lattice of resting or oscillating particles interacting with the fluid particles through a (e.g. Lennard-Jones) potential. The same kind of interaction happens between fluid particles. It is impossible here to review the relevant literature on MD and we refer e.g. to [4, 6, 7, 14, 15] and to references therein. Our approach, being based on kinetic theory, is mesoscopic, in the same spirit of [3] where the Lattice Boltzmann Equation is considered.

Our approach is based on the idea of considering the wall as a lattice of fixed particles through which fluid particle can penetrate to be specularly reflected by the inner layer of the wall. The penetration probability is ruled by the Poisson distribution. Moreover, as typical in the classical Maxwell kernel, we allow a portion of the particles to thermalize with the wall. The interaction is described by a scattering kernel whose expression is given in (4.4), and which represents a generalization of the classical Maxwell kernel.

The boundary conditions derived in the hydrodynamical limit for the macroscopic velocity do not contain non-local terms: the tangential component of the velocity at the boundary satisfy a Robin boundary condition. On the other hand the boundary condition for the temperature is given by a Robin boundary condition plus an extra term (weakly non local) which is proportional to the divergence of the tangential component of the velocity.

The plan of the paper is the following: in Sect. 2 we introduce some basic notation. In Sect. 3 the basic requirements of the scattering kernel are briefly recalled. In Sect. 4 we propose our model scattering kernel, while in Sect. 5 we study the asymptotic limit for small Knudsen number and we derive the boundary conditions for the fluid-dynamic variables. The setting we shall adopt for this analysis is the Sone's (or modified Hilbert's) expansion [22]. Finally, for the reader's convenience, two appendices are inserted. In Appendix 1 the definition and the basic properties of the collision integral are given. These properties are used for the Knudsen layer analysis. In Appendix 2 the explicit expressions of the first and second order Hilbert solution are given. 


\section{Notation}

We shall be concerned with the mathematical description of the behavior of a fluid composed by a collection of identical point particles confined to a spatial domain $\Omega=\mathbb{R}_{+} \times \mathbb{R}^{2}$. Let us introduce some notation: the $\boldsymbol{X}=X_{i}(i=1,2,3)$ are the Cartesian coordinates of the physical space, $\hat{X}_{1}$ is the unit vector normal to the boundary wall, $\xi=\left(\xi_{1}, \xi_{2}, \xi_{3}\right)$ is the molecular velocity; $f(\boldsymbol{X}, \boldsymbol{\xi})$ is the velocity distribution function of the gas molecules; $\rho$ is the density, $T$ is the temperature, $p$ is the pressure of the gas and $R$ is the gas constant per unit mass. We also introduce the corresponding dimensionless quantities: let $L, p_{0}, T_{0}$ be, respectively, the reference length, pressure and temperature and set $\rho_{0}=p_{0} /\left(R T_{0}\right)^{-1}$, then:

$$
\begin{aligned}
& x_{i}=X_{i} / L, \quad \zeta_{i}=\xi_{i} /\left(2 R T_{0}\right)^{1 / 2}, \quad \hat{f}=f /\left[\rho_{0}\left(2 R T_{0}\right)^{-1}\right], \\
& \hat{\rho}=\rho / \rho_{0}, \quad u_{i}=v_{i} /\left(2 R T_{0}\right)^{1 / 2}, \quad \hat{p}=p / p_{0}, \quad \hat{T}=T / T_{0}, \\
& \hat{T}_{w}=T_{w} / T_{0}, \quad \hat{\rho}_{w}=\rho_{w} / \rho_{0}, \quad \hat{p}_{w}=p_{w} / p_{0},\left(\hat{p}_{w}=\hat{\rho}_{w} \hat{T}_{w}\right),
\end{aligned}
$$

where $v_{i}$ are the components of the gas velocity and $T_{w}, \rho_{w}$ and $p_{w}$ are the wall temperature, density and pressure, respectively.

We also introduce the following notation:

$$
E(\zeta)=\frac{1}{\pi^{3 / 2}} \exp \left(-\zeta^{2}\right), \quad \zeta=\left(\zeta_{i}^{2}\right)^{1 / 2}=\left|\zeta_{i}\right|
$$

so that the Maxwellian distribution $f_{0}$ with $v_{i}=0, p=p_{0}$ and $T=T_{0}$ :

$$
f_{0}=\frac{\rho_{0}}{\left(2 \pi R T_{0}\right)^{3 / 2}} \exp \left(-\frac{\xi_{i}^{2}}{2 R T_{0}}\right)
$$

can be written in the form:

$$
f_{0}=\frac{\rho_{0}}{\left(2 \pi R T_{0}\right)^{3 / 2}} E(\zeta)
$$

In what follows we shall consider the state of the gas to be close to an equilibrium state at rest, which choose to be the Maxwellian distribution function $f_{0}$ given by (2.4). The nondimensional perturbed variables are given by:

$$
\begin{aligned}
& \phi=\left\{\begin{array}{l}
f / f_{0}-1, \\
\hat{f} / E-1,
\end{array}\right. \\
& \omega=\left\{\begin{array}{l}
\rho / \rho_{0}-1, \\
\hat{\rho}-1
\end{array}\right. \\
& \tau=\left\{\begin{array}{l}
T / T_{0}-1, \\
\hat{T}-1,
\end{array}\right. \\
& P=\left\{\begin{array}{l}
p / p_{0}-1, \\
\hat{p}-1,
\end{array}\right. \\
& \tau_{w}=\left\{\begin{array}{l}
T_{w} / T_{0}-1, \\
\hat{T}_{w}-1
\end{array}\right. \\
& \omega_{w}=\left\{\begin{array}{l}
\rho_{w} / \rho_{0}-1, \\
\hat{\rho}_{w}-1,
\end{array}\right. \\
& P_{w}=\left\{\begin{array}{l}
p_{w} / p_{0}-1, \\
\hat{p}_{w}-1 .
\end{array}\right. \\
& P_{w}=\omega_{w}+\tau_{w}+\omega_{w} \tau_{w},
\end{aligned}
$$

Then the steady Boltzmann equation in dimensionless form reads:

$$
\zeta_{i} \frac{\partial \phi}{\partial x_{i}}=\frac{1}{k}[\mathcal{L}(\phi)+\mathcal{J}(\phi, \phi)],
$$


where $\mathcal{J}(\phi)$ and $\mathcal{L}(\phi)$ are the collision integral and the linearized collision integral, respectively, whose explicit expressions are given in Appendix 1;

$$
k=\frac{\sqrt{\pi} \lambda}{2 L}=\frac{\sqrt{\pi}}{2} \mathrm{Kn},
$$

$\mathrm{Kn}$ is the Knudsen number and $\lambda$ is the mean free path of the gas molecules.

Throughout the rest of this paper we shall make use of the following notation for the local Maxwellian distribution in the nondimensional perturbed form:

$$
E \phi_{e}\left(\omega, u_{i}, \tau\right)=\frac{1+\omega}{\pi^{3 / 2}(1+\tau)^{3 / 2}} \exp \left(-\frac{\left(\zeta_{i}-u_{i}\right)^{2}}{1+\tau}\right)-E,
$$

which satisfies:

$$
\mathcal{L}\left(\phi_{e}\right)+\mathcal{J}\left(\phi_{e}, \phi_{e}\right)=0
$$

The relation of the nondimensional macroscopic variables and the nondimensional velocity distribution function $\phi$ are:

$$
\left\{\begin{array}{l}
\omega=\int \phi E d \zeta \\
(1+\omega) u_{i}=\int \zeta_{i} \phi E d \zeta \\
\frac{3}{2}(1+\omega) \tau=\int\left(\zeta_{i}^{2}-\frac{3}{2}\right) \phi E d \zeta-(1+\omega) u_{i}^{2} \\
P=\omega+\tau+\omega \tau
\end{array}\right.
$$

\section{Gas-Surface Interaction and Scattering Kernel Requirements}

Let us consider a particle hitting the wall at $x_{1}=0$ : let $\zeta^{\prime}=\left(\zeta_{1}^{\prime}, \zeta_{2}^{\prime}, \zeta_{3}^{\prime}\right)$ be the velocity of the impinging particle referred to the wall and $\zeta=\left(\zeta_{1}, \zeta_{2}, \zeta_{3}\right)$ is the velocity of the reflected particle. Moreover let $\boldsymbol{y}=\left(x_{2}, x_{3}\right)$ be the position of a point on the plane $x_{1}=0$.

In what follows we shall always deal with the case of a simple boundary, namely a wall where there is no mass flux across it.

Due to the very complicated mechanisms that take place between the surface layers of the solid wall and the interaction potential of the gas molecules, one usually assumes a quite general gas-surface interaction law of the following form:

$$
\begin{aligned}
&|\boldsymbol{\zeta} \cdot \boldsymbol{n}| E(\zeta)(1+\phi(\boldsymbol{y}, \zeta)) \\
& \quad=\int_{\zeta^{\prime} \cdot \boldsymbol{n}<0}\left|\boldsymbol{\zeta}^{\prime} \cdot \boldsymbol{n}\right| R\left(\boldsymbol{\zeta}^{\prime} \rightarrow \zeta ; \boldsymbol{y}\right) E\left(\zeta^{\prime}\right)\left(1+\phi\left(\boldsymbol{y}, \boldsymbol{\zeta}^{\prime}\right)\right) d \zeta^{\prime} \quad\left(x_{1}=0, \boldsymbol{\zeta} \cdot \boldsymbol{n}>0\right),
\end{aligned}
$$

where $\boldsymbol{n}$ is the unit vector normal to the boundary and $R\left(\zeta^{\prime} \rightarrow \zeta ; \boldsymbol{y}\right)$ is the scattering kernel, i.e. the probability that a molecule impinging the wall at point $y$ with velocity $\zeta^{\prime}$ is scattered with velocity between $\zeta$ and $\zeta+d \zeta$. The problem of writing good boundary conditions for the Boltzmann equation is to write a scattering kernel which correctly reproduces the phenomena occurring at the wall.

Any physical kernel has to satisfy some conditions. Firstly, as it is a probability density,

$$
R\left(\zeta^{\prime} \rightarrow \zeta ; y\right) \geq 0
$$


must hold. The conservation of the mass at the wall (nonabsorbing and nonporous) is expressed by the following relation:

$$
\int_{\zeta \cdot n>0} R\left(\zeta^{\prime} \rightarrow \zeta ; \boldsymbol{y}\right) d \zeta=1 \quad\left(\boldsymbol{\zeta}^{\prime} \cdot \boldsymbol{n}<0\right),
$$

which, in turn, implies that the normal component of the velocity at the wall must be zero (see Chap. III of [5]).

The last condition is the so-called Detailed Balance or Reciprocity relation, namely:

$$
\left|\boldsymbol{\zeta}^{\prime} \cdot \boldsymbol{n}\right| E\left[1+\phi_{e}\left(\boldsymbol{\zeta}^{\prime}\right)\right] R\left(\boldsymbol{\zeta}^{\prime} \rightarrow \boldsymbol{\zeta} ; \boldsymbol{y}\right)=|\boldsymbol{\zeta} \cdot \boldsymbol{n}| E\left[1+\phi_{e}(-\zeta)\right] R\left(-\boldsymbol{\zeta} \rightarrow-\boldsymbol{\zeta}^{\prime} ; \boldsymbol{y}\right),
$$

where $\phi_{e}$ is the Maxwellian given by (2.8). It can be formulated as follows: at the equilibrium the number of particles hitting the wall with velocity $\zeta^{\prime}$ and scattered with velocity $\zeta$ at point $\boldsymbol{y}$ is equal to the number of particle hitting with velocity $-\zeta$ and scattered with velocity $-\zeta^{\prime}$ at the same point $\boldsymbol{y}$.

The theoretical modeling of the gas-surface interaction was first investigated by Maxwell. He proposed a scattering kernel of the following form:

$$
\begin{aligned}
& R\left(\boldsymbol{\zeta}^{\prime} \rightarrow \boldsymbol{\zeta}, \boldsymbol{y}\right)=(1-\alpha) \delta\left(\zeta_{1}+\zeta_{1}^{\prime}\right) \delta\left(\zeta_{2}-\zeta_{2}^{\prime}\right) \delta\left(\zeta_{3}-\zeta_{3}^{\prime}\right) \\
&+\alpha \frac{2}{\pi\left(\hat{T}_{w}\right)^{2}} \zeta_{1} \exp \left[-\frac{\left(\zeta_{i}-u_{i w}\right)^{2}}{\hat{T}_{w}}\right], \\
& \text { for } \zeta_{1}^{\prime}<0, \zeta_{1}>0
\end{aligned}
$$

where $u_{i w}$ is the wall velocity. The above model prescribes that an $1-\alpha$ fraction of the molecules is specularly reflected at the surface of the wall, while the remaining $\alpha$ fraction of the molecules is in thermal equilibrium with the wall: it gets absorbed and re-emitted after a multiple-collision interaction with the molecules of the wall lattice. The case $\alpha=1$ corresponds to the diffuse-reflection boundary condition and the case $\alpha=0$ gives perfect specular reflection. All the interactions are local in space: the particle hitting at point $\boldsymbol{y}$ of the wall is re-emitted at the same position.

\section{Nonlocal Scattering Kernel}

In this section we want to propose a different model of the interaction between the fluid particles and the wall. We suppose that, other than being reflected at the surface of the wall, the fluid molecules have a non-zero probability of passing some layers of the wall without experiencing any impact and then of being specularly reflected by some inner molecule of the wall lattice. This introduces a nonlocality effect into the scattering kernel: in fact if the molecule hits the wall at point $\boldsymbol{y}^{\prime}$ on the wall $x_{1}=0$, it will travel for some distance inside the wall, will strike the lattice and will come out at a different point $\boldsymbol{y}$. Since it is specularly reflected, the impact will take place half-way between $\boldsymbol{y}^{\prime}$ and $\boldsymbol{y}$.

Let us first introduce the following notation. Let

$$
\begin{aligned}
& \boldsymbol{y}=\left(x_{2}, x_{3}\right), \quad \boldsymbol{y}-\boldsymbol{y}^{\prime}=\rho_{y}\left(\begin{array}{c}
\cos \alpha_{y} \\
\sin \alpha_{y}
\end{array}\right), \\
& \zeta_{y}=\left(\zeta_{2}, \zeta_{3}\right)=\rho_{\zeta}\left(\begin{array}{c}
\cos \alpha_{\zeta} \\
\sin \alpha_{\zeta}
\end{array}\right), \quad \zeta_{y}^{\prime}=\left(\zeta_{2}^{\prime}, \zeta_{3}^{\prime}\right)=\rho_{\zeta}^{\prime}\left(\begin{array}{c}
\cos \alpha_{\zeta^{\prime}} \\
\sin \alpha_{\zeta^{\prime}}
\end{array}\right),
\end{aligned}
$$


where $\left(\rho_{y}, \alpha_{y}\right)$ are the polar coordinates on the wall plane $\left(x_{2}, x_{3}\right)$ centered in $\boldsymbol{y}^{\prime}$, and $\left(\rho_{\zeta}, \alpha_{\zeta}\right)$ are the polar coordinates on the wall plane $\left(\zeta_{2}, \zeta_{3}\right)$.

Let $\boldsymbol{\zeta}_{y}^{\prime}$ be the tangential velocity of the incident particle. If $\boldsymbol{y}-\boldsymbol{y}^{\prime}$ is parallel to $\boldsymbol{\zeta}_{y}^{\prime}$ then the probability of the process described above is the product of three different probabilities: the first is the probability of the particle traveling for a distance $\rho_{y} / 2=\left(\left|\boldsymbol{y}-\boldsymbol{y}^{\prime}\right|\right) / 2$ without hitting any other molecule. The second is the probability of having an impact between $\rho_{y} / 2$ and $\rho_{y} / 2+d \rho_{y}$. The third is the probability of traveling again for $\rho_{y} / 2$ without impacts. We shall assume each process to be governed by a Poisson distribution function with $1 / \eta$ as mean value, where $\eta$ is a parameter measuring the rarefaction of the wall (or the strength of the interaction between the wall and the fluid molecules) to be chosen later.

Therefore the above mentioned probabilities are: the first one $\exp \left(-\rho_{y} /(2 \eta)\right)$, the second $d \rho_{y} / \eta$, and the third $\exp \left(-\rho_{y} /(2 \eta)\right)$.

When $\boldsymbol{y}-\boldsymbol{y}^{\prime}$ is not parallel to $\boldsymbol{\zeta}_{y}^{\prime}$ then the probability is 0 . This introduces a Dirac $\delta$ in the angular variable, i.e. $\delta\left(\left(\alpha_{y}-\alpha_{\zeta^{\prime}}\right) / \rho_{y}\right.$ (the factor $\rho_{y}^{-1}$ is due to the use of polar coordinates) which has to multiply the volume element in $\alpha_{\zeta^{\prime}}$ i.e. $\rho_{y} d \alpha_{\zeta^{\prime}}$. Finally one can write:

Knowing that the particles hits the wall at $\boldsymbol{y}^{\prime}$ with velocity $\boldsymbol{\zeta}^{\prime}$, the probability of having just an impact inside the wall and getting out at the volume element $d \rho_{y} d \alpha_{y}$ $\simeq \frac{1}{\eta} \exp \left(-\frac{\rho_{y}}{\eta}\right) \delta\left(\alpha_{y}-\alpha_{\zeta^{\prime}}\right) d \rho_{y} d \alpha_{y}$ centered at $\boldsymbol{y}$ with velocity $\zeta$, is:

This term will affect the specular reflection part of the scattering kernel. Notice how we are taking as the parameter of the distribution function the quantity $\left|\boldsymbol{y}-\boldsymbol{y}^{\prime}\right|$, instead of the actual distance traveled by the particle inside the wall. This is a simplifying assumption (that at this level could be easily relaxed) that will make manageable the asymptotic analysis in the fluid dynamic limit.

On the other hand, we suppose that the molecules which experience multiple scattering inside the solid will get in thermal and mechanical equilibrium with the wall, so that, as in the Maxwell scattering kernel, they will obey the diffuse reflection law at the boundary. Hence the nonlocal scattering kernel which takes into account both the nonlocal specular reflection and the diffuse reflection is given by:

$$
\begin{aligned}
R\left(\boldsymbol{\zeta}^{\prime} \rightarrow \zeta, \boldsymbol{y}^{\prime} \rightarrow \boldsymbol{y}\right)= & (1-\alpha) \delta\left(\zeta_{1}+\zeta_{1}^{\prime}\right) \delta\left(\zeta_{y}-\zeta_{y}^{\prime}\right) \frac{1}{\eta} e^{-\frac{\left|y-y^{\prime}\right|}{\eta}} \frac{\delta\left(\alpha_{y}-\alpha_{\zeta}^{\prime}\right)}{\rho_{y}} \\
& +\alpha \frac{2}{\pi\left(1+\tau_{w}\right)^{2}} \zeta_{1} \exp \left[-\frac{\left(\zeta_{i}-u_{i w}\right)^{2}}{1+\tau_{w}}\right] \delta\left(\boldsymbol{y}-\boldsymbol{y}^{\prime}\right)
\end{aligned}
$$

for $\zeta_{1}^{\prime}<0, \zeta_{1}>0$

In the above equation (4.4) the constants are chosen so as to satisfy the following nonlocal normalization condition:

$$
\int_{\mathbb{R}^{2}} d \boldsymbol{y} \int_{\zeta_{1}>0} R\left(\zeta^{\prime} \rightarrow \zeta ; \boldsymbol{y}^{\prime} \rightarrow \boldsymbol{y}\right) d \zeta=1 \quad\left(\zeta_{1}^{\prime}<0\right)
$$

Notice that the above condition (4.5) is the nonlocal analog of (3.3): the latter expresses a pointwise conservation of the mass while the former requires the overall mass flux to be conserved. It is obvious that the above scattering kernel satisfies the positivity condition 
(3.2). On the other hand one can easily verify that the reciprocity relation (3.4) is satisfied in the following form:

$$
\begin{aligned}
& \left|\zeta_{1}^{\prime}\right| E\left(\zeta^{\prime}\right)\left[1+\phi_{e}\left(\zeta^{\prime}\right)\right] R\left(\zeta^{\prime} \rightarrow \zeta ; \boldsymbol{y}^{\prime} \rightarrow \boldsymbol{y}\right) \\
& \quad=\left|\zeta_{1}\right| E(\zeta)\left[1+\phi_{e}(-\zeta)\right] R\left(-\zeta \rightarrow-\zeta^{\prime} ; \boldsymbol{y} \rightarrow \boldsymbol{y}^{\prime}\right),
\end{aligned}
$$

where $\phi_{e}$ is the Maxwellian given by (2.8). It can be formulated as follows: at equilibrium the number of particles hitting the wall at point $\boldsymbol{y}^{\prime}$ with velocity $\zeta^{\prime}$ and scattered with velocity $\zeta$ at point $\boldsymbol{y}$ is equal to the number of particle hitting with velocity $-\zeta$ at point $\boldsymbol{y}$ and scattered with velocity $-\boldsymbol{\zeta}^{\prime}$ at the point $\boldsymbol{y}^{\prime}$.

\subsection{Boundary Conditions for the Distribution Function}

We want to find the boundary condition for $\phi$ at the wall corresponding to the scattering kernel $R\left(\boldsymbol{\zeta}^{\prime} \rightarrow \zeta ; \boldsymbol{y}^{\prime} \rightarrow \boldsymbol{y}\right)$ given by (4.4). We impose a nonlocal analog of (3.1), namely we require at the wall the following relation to hold:

$$
\begin{aligned}
& \left|\zeta_{1}\right| E[1+\phi(\boldsymbol{y}, \zeta)] \\
& \quad=\int d \boldsymbol{y}^{\prime} \int_{\zeta_{1}^{\prime}<0}\left|\zeta_{1}^{\prime}\right| R\left(\boldsymbol{\zeta}^{\prime} \rightarrow \boldsymbol{\zeta} ; \boldsymbol{y}^{\prime} \rightarrow \boldsymbol{y}\right) E\left[1+\phi\left(\boldsymbol{y}^{\prime}, \boldsymbol{\zeta}^{\prime}\right)\right] d \boldsymbol{\zeta}^{\prime} \quad\left(x_{1}=0, \boldsymbol{\zeta} \cdot \boldsymbol{n}>0\right),
\end{aligned}
$$

Using the expressions given by (4.4) in (4.7) one gets the following boundary condition in $x_{1}=0$ :

$$
\begin{aligned}
& \phi(\boldsymbol{\zeta}, \boldsymbol{y})=(1-\alpha) \int_{0}^{\infty} d \rho_{y} \frac{1}{\eta} e^{-\frac{\rho_{y}}{\eta}} \phi\left(-\zeta_{1}, \zeta_{y}, \boldsymbol{y}-\rho_{y}\left(\begin{array}{c}
\cos \alpha_{\zeta} \\
\sin \alpha_{\zeta}
\end{array}\right)\right)+\alpha \phi_{e}\left(\check{\sigma_{w}}, u_{w}, \tau_{w}\right)(\boldsymbol{y}, \zeta) \\
& \text { for } \zeta_{1}^{\prime}<0, \zeta_{1}>0
\end{aligned}
$$

where $\phi_{e}\left(\check{\sigma_{w}}, u_{w}, \tau_{w}\right)$ are given by:

$$
\begin{aligned}
& E \phi_{e}\left(\check{\sigma}_{w}, u_{w}, \tau_{w}\right)=\frac{1+\check{\sigma}_{w}}{\pi^{3 / 2}\left(1+\tau_{w}\right)^{3 / 2}} \exp \left(-\frac{\left(\zeta_{i}-u_{i w}\right)^{2}}{1+\tau_{w}}\right)-E, \\
& \check{\sigma}_{w}=-2\left(\frac{\pi}{1+\tau_{w}}\right)^{1 / 2} \int_{\zeta_{1}<0} \zeta_{1} E(\zeta) \phi\left(\zeta_{i}, x_{2}\right) d \zeta+\left(\frac{1}{1+\tau_{w}}\right)^{1 / 2}-1 .
\end{aligned}
$$

\section{The Asymptotic Analysis for Small Knudsen Number}

The aim of this Section is to analyze the limit $\mathrm{Kn} \rightarrow 0$ starting from the kinetic description given by (2.6) with the boundary condition (4.8). Our aim is to get the set of boundary conditions for the fluid dynamic variables in the leading order, namely $O(\mathrm{Kn})$. We shall consider the case where the accommodation coefficient is of the same order of magnitude of the Knudsen number. That is, we set:

$$
\begin{aligned}
\varepsilon & =\frac{\sqrt{\pi}}{2} \mathrm{Kn}(=k) \ll 1, \\
\alpha & =\beta \varepsilon,
\end{aligned}
$$


where $\beta$ is a given constant and $\varepsilon$ is a small parameter. The case where the accommodation coefficient $\alpha=O(\varepsilon)$, was tackled in [1] where, on the basis of the linearized BKW equation with an arbitrary but smooth shaped boundary, the Stokes system with mixed-type boundary conditions was derived. Recently the small accommodation coefficient case was analyzed in [2], where the authors studied the cylindrical Couette flow of a rarefied gas using the nonlinear Boltzmann equation.

We shall choose:

$$
\eta=a \varepsilon, \quad a>0,
$$

which means that the mean free flight of the fluid particles inside the wall is of the same order of the mean free path inside the fluid.

The asymptotic analysis of the boundary value problem (2.6) and (4.8), together with (5.1-5.2), will give, to the leading order, the set of Navier-Stokes equations and its appropriate boundary conditions. Since the procedure to be carried out in order to derive the fluid dynamic equations is described in detail in the book of Sone [22], here we shall only briefly outline the main steps.

We shall investigate the asymptotic behavior of the solution of the boundary value problem (2.6) and (4.8) when $\mathrm{Kn} \rightarrow 0$. We want to describe the case of finite Reynolds number, namely $\operatorname{Re}=O(1)$, so that, according to the Von Karmann relation (Ma $\propto \mathrm{ReKn})$, the deviation of the system from a uniform equilibrium state at rest has to be of the same order as $\mathrm{Kn}$. This accounts to choose $\phi=O(\varepsilon)$. In terms of the macroscopic parameters, the assumption on the velocity distribution function requires that the nondimensional temperature and density variation are $O(\varepsilon)$.

First, putting aside the boundary condition (4.8), we look for a moderately varying solution of (2.6) (which we shall denote by $\phi_{S}$ ), in the form of a power series of $\varepsilon$, which satisfies $\partial \phi_{S} / \partial x_{i}=O\left(\phi_{S}\right)$ :

$$
\phi_{S}=\phi_{S 1} \varepsilon+\phi_{S 2} \varepsilon^{2}+\cdots,
$$

where the series starts from the first order in $\varepsilon$ since $\phi$ is assumed to be $O(\varepsilon)$, and the component function $\phi_{S m}$ is a quantity of order of unity. This $\phi_{S}$ is called the $S$ (or Hilbert) solution.

The relation between the macroscopic variables and the distribution function is the same as (2.10), except for the subscript $S$.

Corresponding to the expansion (5.4), the macroscopic variables $\omega_{S}, u_{i S}, \tau_{S}, \ldots$ are also expanded in $\varepsilon$ :

$$
h_{S}=h_{S 1} \varepsilon+h_{S 2} \varepsilon^{2}+\cdots,
$$

where $h$ represents $\omega, u_{i}, \tau, P$ and the component function $h_{S m}$ is a quantity of the order of unity. The relation between the component function $h_{S m}$ of the macroscopic variable $h_{S}$ and the component function $\phi_{S r}$ is obtained by substituting in (2.10), (5.4) and (5.5) and by equating the coefficients of the same power of $\varepsilon$.

Substituting the expansion (5.4) into the Boltzmann equation (2.6) and arranging the same order quantities in $\varepsilon$, we obtain a sequence of integral equations for $\phi_{S m}$, which can be solved from the lowest order. For the leading order we get $\mathcal{L}\left(\phi_{S 1}\right)=0$, whose solution is given by a local Maxwellian distribution.

As for the higher order terms, one gets the inhomogeneous Boltzmann equation, namely a linear integral equation containing the linearized collision integral $\mathcal{L}$, with inhomogeneous terms consisting of the earlier terms of the expansion. The corresponding homogeneous equation, namely $\mathcal{L}\left(\phi_{S m}\right)=0$, has five independent solutions $1, \zeta_{i}, \zeta_{i}^{2}$. This implies that, for 
the inhomogeneous equation to have a solution, its inhomogeneous term should satisfy the solvability conditions:

$$
\int g \zeta_{i} \frac{\partial \phi_{S m-1}}{\partial x_{i}} E d \zeta=0
$$

where $g=1, \zeta_{i}$, or $\zeta_{i}^{2}$. The application of this condition to $\phi_{S 1}, \phi_{S 2}, \ldots$ in the solution process of the sequence of the integral equations from the lower order, leads to the fluid dynamic equations for $h_{m}$ in (5.5).

The explicit expressions of $\phi_{S 1}$ and $\phi_{S 2}$ are explicitly given in Appendix 2; rearranging the equations obtained from the series of the solvability conditions (5.6), one gets the set of equations that determine the component functions of the expansion of the macroscopic variables:

$$
\begin{aligned}
& \frac{\partial P_{S 1}}{\partial x_{i}}=0, \\
& \frac{\partial u_{i S 1}}{\partial x_{i}}=0, \\
& u_{j S 1} \frac{\partial u_{i S 1}}{\partial x_{j}}=-\frac{1}{2} \frac{\partial P_{S 2}}{\partial x_{i}}+\frac{\gamma_{1}}{2} \frac{\partial^{2} u_{i S 1}}{\partial x_{j}^{2}}, \\
& u_{i S 1} \frac{\partial \tau_{S 1}}{\partial x_{i}}=\frac{\gamma_{2}}{2} \frac{\partial^{2} \tau_{S 1}}{\partial x_{i}^{2}} .
\end{aligned}
$$

where $i, j=1,2,3$, and the expression of $\gamma_{1}$ and $\gamma_{2}$ are given in Appendix 2 .

Equation (5.7) is the momentum equation at the order $\varepsilon$ and imposes $P_{S 1}$ to be a constant. The pressure variation at order $\varepsilon$ must vanish for a flow field with Mach number of order $\varepsilon$ to be established. The next equations (5.8-5.10), which determine $\omega_{S 1}, u_{i S 1}, \tau_{S 1}$ and $P_{S 2}$ are the Navier-Stokes equations for an incompressible fluid, with $\gamma_{1}$ and $\gamma_{2}$ as the nondimensional viscosity and thermal conductivity whose explicit expressions are given in Appendix 2.

We now focus on the derivation of the set of the boundary conditions and therefore look for an asymptotic expansion of the boundary conditions (4.8).

Making the following change of variable $z=\rho_{y} /(a \varepsilon)$ in (4.8), where $a$ is the constant introduced in (5.3), one gets:

$$
\begin{aligned}
& \phi(\boldsymbol{\zeta}, \boldsymbol{y})=(1-\varepsilon \beta) \int_{0}^{\infty} d z e^{-z} \phi\left(-\zeta_{1}, \zeta_{y}, \boldsymbol{y}-a \varepsilon z\left(\begin{array}{c}
\cos \alpha_{\zeta} \\
\sin \alpha_{\zeta}
\end{array}\right)\right)+\varepsilon \beta \phi_{e}\left(\check{\sigma_{w}}, u_{w}, \tau_{w}\right)(\boldsymbol{y}, \zeta) \\
& \quad \text { for } \zeta_{1}^{\prime}<0, \zeta_{1}>0 .
\end{aligned}
$$

If one now expands the function $\phi$ in power series of $\varepsilon$ and retains only the terms $O(\varepsilon)$ (which is sufficient to get the boundary conditions at order $O\left(\varepsilon^{2}\right)$ since $\phi=O(\varepsilon)$ ), the following boundary condition is obtained:

$$
\begin{aligned}
& \phi(\boldsymbol{\zeta}, \boldsymbol{y})=(1-\varepsilon \beta) \phi\left(-\zeta_{1}, \zeta_{y}, \boldsymbol{y}\right)+\varepsilon \beta \phi_{e}\left(\check{\sigma_{w}}, u_{w}, \tau_{w}\right)(\boldsymbol{y}, \zeta) \\
&-\left.a \varepsilon(1-\varepsilon \beta)\left(\begin{array}{c}
\cos \alpha_{\zeta} \\
\sin \alpha_{\zeta}
\end{array}\right) \cdot \frac{\partial \phi}{\partial \boldsymbol{y}}\right|_{\varepsilon=0}\left(-\zeta_{1}, \zeta_{y}, \boldsymbol{y}\right)+O\left(\varepsilon^{2}\right), \\
& \text { for } \zeta_{1}^{\prime}<0, \zeta_{1}>0 .
\end{aligned}
$$


We notice that the first row in (5.12) is the usual Maxwell boundary condition, while the second row is the effect of the nonlocality of the scattering kernel.

In all the above procedure we are considering the steady case. In the unsteady case one cannot ignore the fact that the particle, entering at location $\boldsymbol{y}^{\prime}$ would get out at $\boldsymbol{y}$ with a time delay. If one would consider this effect, then, in the asymptotic expansion (5.12), one would get the time derivative of the $\phi$.

\subsection{Boundary Conditions for the Fluid Dynamic Variables}

We now take into account the boundary conditions (5.12) that were put aside in the previous section when the distribution function $\phi_{S}$ was derived. Since $\phi_{S 1}$ is a local Maxwellian given by (8.1), it can be made so as to satisfy the boundary conditions (5.12) at order $\varepsilon$. In other words, provided that

$$
u_{1 S 1}=u_{1 w 1}=0,
$$

$\phi_{S 1}$ satisfies the boundary conditions in the leading order, i.e.

$$
\phi_{S 1}(\zeta, \boldsymbol{y})=\phi_{S 1}\left(-\zeta_{1}, \zeta_{y}, \boldsymbol{y}\right)
$$

However, the next order term $\phi_{S 2}$, in no longer a Maxwellian (see (8.2)), so that, as in the usual situation, it cannot satisfy the boundary conditions at order $\varepsilon^{2}$. This is not surprising as the Boltzmann equation (2.6) is of singular type. To obtain the solution of the boundary value problem we need to introduce a correction close to the boundary, the so-called Knudsen layer. We shall put the solution in the form:

$$
\phi=\phi_{S}+\phi_{K},
$$

where

$$
\phi_{K}=\varepsilon^{2} \phi_{K 2}+\varepsilon^{3} \phi_{K 3}+\cdots,
$$

is the Knudsen solution, which varies appreciably in a thin layer of thickness $O(\varepsilon)$ adjacent to the boundary $\left(\varepsilon \partial \phi_{K} / \partial x_{1}=O(\varepsilon)\right)$. We stress the fact that the expansion starts form order $\varepsilon^{2}$ because $\phi_{S 1}$ could satisfy the boundary conditions at order $\varepsilon$. Introducing the normal stretched variable $\chi=x_{1} / \varepsilon$, so that $\phi_{K}=\phi_{K}(\zeta, \chi, y)$ and substituting (5.14) into (2.6) with (5.4) and (5.16), one gets the equation and boundary conditions for $\phi_{K 2}$ :

$$
\begin{gathered}
\zeta_{1} \frac{\partial \phi_{K 1}}{\partial \chi}=\mathcal{L}\left(\phi_{K 2}\right) \\
\phi_{K 2}(\boldsymbol{\zeta}, \boldsymbol{y})=-\phi_{S 2}(\boldsymbol{\zeta}, \boldsymbol{y})+\phi_{S 2}\left(-\zeta_{1}, \zeta_{y}, \boldsymbol{y}\right)+\phi_{K 2}\left(-\zeta_{1}, \zeta_{y}, \boldsymbol{y}\right) \\
+\beta\left[\check{\sigma}_{w 1}-\omega_{S 1}+2 \zeta_{i}\left(u_{i w 1}-u_{i S 1}\right)+\left(\zeta_{i}^{2}-\frac{3}{2}\right)\left(\tau_{w 1}-\tau_{S 1}\right)\right] \\
-\left.a\left(\begin{array}{c}
\cos \alpha_{\zeta} \\
\sin \alpha_{\zeta}
\end{array}\right) \cdot \frac{\partial \phi}{\partial \boldsymbol{y}}\right|_{\varepsilon=0}\left(-\zeta_{1}, \zeta_{y}, \boldsymbol{y}\right), \quad \text { for } \zeta_{1}>0 \text { at } \chi=0 .
\end{gathered}
$$

where the macroscopic quantities appearing in $\phi_{e}$ have been expanded in $\varepsilon$ and

$$
\check{\sigma}_{w 1}=\frac{1}{2}\left(\tau_{S 1}-\tau_{w 1}\right)+\omega_{S 1} .
$$


Equations (5.17a-5.17b) are the half-space boundary value problem of the linearized Boltzmann equation.

In order to get the boundary conditions for the fluid dynamic variables we use the orthogonality condition (the analog of (5.6)) for $\phi_{K 2}$ :

$$
\frac{\partial}{\partial \chi} \int g \zeta_{1} \phi_{K 2} E d \zeta=0, \quad \text { for } \chi \geq 0
$$

from which

$$
\int g \zeta_{1} \phi_{K 2} E d \zeta=0, \quad \forall \chi \geq 0
$$

where $g=1, \zeta_{i}$, or $\zeta_{i}^{2}$ are the collision invariants. We now evaluate (5.19) at the boundary $\chi=0$, so that $\phi_{K 2}$ is given by (5.17b), and the explicit expressions of $\phi_{S 1}$ and $\phi_{S 2}$ given in Appendix 2 are used.

When we evaluate (5.19) for $g=1$, we get the following condition:

$$
u_{1 S 2}+\frac{a}{4}\left(\frac{\partial u_{2 S 1}}{\partial x_{2}}+\frac{\partial u_{3 S 1}}{\partial x_{3}}\right)=0 .
$$

This means that at second order in the mean free-path the no-flux condition for the normal velocity is not satisfied. However an integration shows that the zero total flux condition (throughout the whole wall) is satisfied.

When we consider (5.19) for $g=\zeta_{2}$, use the explicit expression for $\phi_{S 1}$ and evaluate the integrals over the velocities, we get:

$$
\sqrt{\pi} \gamma_{1} \frac{\partial u_{2 S 1}}{\partial x_{1}}+2 \beta\left(u_{2 S 1}-u_{2 w 1}\right)=0
$$

Analogously, when one evaluates (5.19) for $g=\zeta_{3}$, one gets:

$$
\sqrt{\pi} \gamma_{1} \frac{\partial u_{3 S 1}}{\partial x_{1}}+2 \beta\left(u_{3 S 1}-u_{3 w 1}\right)=0 .
$$

Finally, evaluating (5.19) for $g=\zeta^{2}$, one obtains the following boundary condition for the temperature:

$$
\gamma_{2} \frac{\partial \tau_{S 1}}{\partial x_{1}}-\frac{4}{5 \sqrt{\pi}} \beta\left(\tau_{S 1}-\tau_{w 1}\right)-\frac{1}{2} a\left(\frac{\partial u_{2 S 1}}{\partial x_{2}}+\frac{\partial u_{3 S 1}}{\partial x_{3}}\right)=0 .
$$

The above condition shows that a tangential divergence of the velocity can create a heat flux through the wall also in absence of thermal gradients. This is an effect (weakly nonlocal) of the non-local character of the fluid-wall interaction.

Equations (5.13), (5.21), (5.22) and (5.23) are the boundary conditions for the fluid dynamic equations (5.7-5.10). Equation (5.13) is the usual no-flux boundary condition. Equations (5.21-5.22) are the Robin boundary conditions for the tangential component of the velocity. Equation (5.23) is the usual Robin boundary condition for the temperature plus an extra term which is proportional to the tangential divergence of the velocity. 


\section{Concluding Remarks}

In this paper we have considered the possibility of non-local interaction of the fluid particle with the wall molecules. The non-locality arose as the consequence of the possibility of the penetration of the fluid particles through the wall lattice followed by a specular reflection. The model resulted of in a non-local scattering kernel, whose expression is given in (4.4).

In the hydrodynamic limit we assumed the penetration length inside the wall to be of the same order of the mean free path inside the fluid. The Knudsen layer analysis led to a set of boundary conditions for the Navier-Stokes equations: (5.13) which is the usual no-flux boundary condition, (5.21-5.22) which are the classical Robin boundary conditions for the tangential velocity, and (5.23) for the temperature flux where the effect of the non-locality shows as a heat flux induced by the velocity gradients.

A similar analysis starting from more realistic gas-surface interaction models could also be of interest. In fact, the criticisms opposed to the Maxwell scattering kernel [8, 17-19] suggest to take into account the case when more than one accommodation coefficient is present (see the Cercignani-Lampis model [8-10], and the anisotropic scattering kernel [12]). This will be the subject of a subsequent work.

Acknowledgements The work of the authors (M.C.L.) and (M.S.) was supported by the INDAM, under the 2005-2007 PRIN grant "Nonlinear Propagation and Stability in Thermodynamical Processes of Continuous Media". They also thank C. Cercignani and A. Majorana for the enlightening discussions on the topics of this paper.

\section{Appendix 1: The Collision Integral}

The collision integral $\mathcal{J}(\phi, \psi)$ is given by:

$$
\begin{aligned}
& \mathcal{J}(\phi, \psi)=\frac{1}{2} \int E_{*}\left(\phi^{\prime} \psi_{*}^{\prime}+\phi_{*}^{\prime} \psi^{\prime}-\phi \psi_{*}-\phi_{*} \psi\right) \hat{B} d \Omega(\boldsymbol{\alpha}) d \zeta_{*}, \\
& \phi=\phi\left(\zeta_{i}\right), \quad \phi_{*}=\phi\left(\zeta_{i *}\right), \quad \phi^{\prime}=\phi\left(\zeta_{i}^{\prime}\right), \quad \phi_{*}^{\prime}=\phi\left(\zeta_{i *}^{\prime}\right),
\end{aligned}
$$

where $\zeta_{i}^{\prime}$ and $\zeta_{i *}^{\prime}$ are related to $\zeta_{i}$ and $\zeta_{i *}$ by

$$
\zeta_{i}^{\prime}=\zeta_{i}+\alpha_{i} \alpha_{j}\left(\zeta_{j *}-\zeta_{j}\right), \quad \zeta_{i *}^{\prime}=\zeta_{i}+\alpha_{i *} \alpha_{j}\left(\zeta_{j *}-\zeta_{j}\right)
$$

$\alpha_{i}$ is a unit vector expressing the variation of the direction of the molecular velocity owing to an intermolecular collision, $d \Omega(\boldsymbol{\alpha})$ is the solid-angle element in the direction of $\alpha_{i}$ and $\hat{B}=\hat{B}\left(\left|\alpha_{i}\left(\zeta_{i *}-\zeta_{i}\right)\right| /\left|\left(\zeta_{k *}-\zeta_{k}\right)\right|,\left|\left(\zeta_{i *}-\zeta_{i}\right)\right|\right)$, generally depends on $T_{0}$ as well as on the intermolecular potential. For hard-sphere gas

$$
\hat{B}=\frac{\left|\alpha_{i}\left(\zeta_{i *}-\zeta_{i}\right)\right|}{4(2 \pi)^{1 / 2}}
$$

$d \zeta_{*}=d \zeta_{* 1} d \zeta_{* 2}$; the domain of integration in (7.1a) is all directions of $\boldsymbol{\alpha}$ and the whole space of $d \zeta_{*}$ (see Sect. 2.9 in [22]).

The linear part of the collision integral, called the linearized collision integral $\mathcal{L}(\phi)$ is given by:

$$
\mathcal{L}(\phi)=\int E_{*}\left(\phi^{\prime}+\phi_{*}^{\prime}-\phi-\phi_{*}\right) \hat{B} d \Omega(\boldsymbol{\alpha}) d \zeta_{*}
$$


The operator $\mathcal{J}$ is related to $\mathcal{L}$ in the following way:

$$
2 \mathcal{J}(1, \phi)=\mathcal{L}(\phi) .
$$

Moreover the operator $\mathcal{L}$ satisfies the following relations:

$$
\begin{aligned}
& \int E \psi(\zeta) \mathcal{L}(\phi) d \zeta=\frac{1}{4} \int E E_{*}\left(\psi+\psi_{*}-\psi^{\prime}-\psi_{*}^{\prime}\right)\left(\phi^{\prime}+\phi_{*}^{\prime}-\phi-\phi_{*}\right) \hat{B} d \Omega(\boldsymbol{\alpha}) d \zeta_{*} d \zeta, \\
& \text { for any } \phi \text { and } \psi, \\
& \int g(\zeta) \mathcal{L}(\phi) E d \zeta=0 \quad \text { for any } \phi, \\
& \mathcal{L}(g(\zeta))=0,
\end{aligned}
$$

where $g(\zeta)$ is one of the collision invariants $1, \zeta_{i}$, or $\zeta_{i}^{2}$. Moreover, from (7.5), it follows that the operator $\mathcal{L}$ is selfadjoint. Finally:

$$
\begin{array}{cl}
\int g(\zeta) \mathcal{J}(\phi, \psi) E d \zeta=0 & \text { for any } \phi \text { and } \psi \\
\int g(\zeta) \mathcal{L}(\phi) E d \zeta=0 & \text { for any } \phi
\end{array}
$$

\section{Appendix 2: Explicit Expressions of $\phi_{S 1}$ and $\phi_{S 2}$}

The first-order S-solution $\phi_{S 1}$ is given by:

$$
\phi_{S 1}=\omega_{S 1}+2 \zeta_{i} u_{i S 1}+\left(\zeta_{i}^{2}-\frac{3}{2}\right) \tau_{S 1}
$$

This is the first term of the expansion of the perturbed Maxwellian $\phi_{e}\left(\omega_{S}, u_{i S}, \tau_{S}\right)\left(=\phi_{e S}\right)$ in terms of $\varepsilon$.

The second-order S-solution $\phi_{S 2}$ is given by:

$$
\phi_{S 2}=\phi_{e S 2}-\zeta_{i} \zeta_{j} B(\zeta) \frac{\partial u_{i S 1}}{\partial x_{j}}-\zeta_{i} A(\zeta) \frac{\partial \tau_{S 1}}{\partial x_{i}},
$$

where $\phi_{e S 2}$ is the second term of the expansion of the perturbed Maxwellian $\phi_{e S}$ and the functions $A(\zeta)$ and $B(\zeta)$ are the solutions of the following integral equations:

$$
\begin{aligned}
& \left\{\begin{array}{l}
\mathcal{L}\left[\zeta_{i} A(\zeta)\right]=-\zeta_{i}\left(\zeta_{j}^{2}-\frac{5}{2}\right), \\
\text { subsidiary condition } \int_{0}^{\infty} \zeta^{4} A(\zeta) \exp \left(-\zeta^{2}\right) d \zeta=0,
\end{array}\right. \\
& \mathcal{L}\left[\left(\zeta_{i} \zeta_{j}-\frac{1}{3} \zeta_{k}^{2} \delta_{i j}\right) B(\zeta)\right]=-2\left(\zeta_{i} \zeta_{j}-\frac{1}{3} \zeta_{k}^{2} \delta_{i j}\right)
\end{aligned}
$$

The coefficients $\gamma_{1}$ and $\gamma_{2}$ occurring in the Navier-Stokes equations (5.8-5.10) are given by:

$$
\gamma_{1}=I_{6}(B), \quad \gamma_{2}=2 I_{6}(A), \quad I_{n}(Z)=\frac{8}{15 \sqrt{\pi}} \int_{0}^{\infty} \zeta^{n} Z(\zeta) \exp \left(-\zeta^{2}\right) d \zeta
$$




\section{References}

1. Aoki, K., Inamuro, T., Onishi, Y.: Slightly raefied gas flow over a body with small accommodation coefficient. J. Phys. Soc. Jpn. 47, 663-671 (1979)

2. Aoki, K., Yoshida, H., Nakanishi, T., Garcia, A.L.: Inverted velocity profile in the cylindrical couette flow of a rarefied gas. Phys. Rev. E 68, 016302 (2003)

3. Benzi, R., Biferale, L., Sbragaglia, M., Succi, S., Toschi, F.: Mesoscopic modeling of heterogeneous boundary conditions for microchannel flows. J. Fluid Mech. 548, 257-280 (2006)

4. Cottin-Bizonne, C., Barentin, C., Charlaix, É., Bocquet, L., Barrat, J.-L.: Dynamics of simple liquids at heterogeneous surfaces: molecular-dynamics simulations and hydrodynamic description. Eur. Phys. J. E 15, 427-438 (2004)

5. Cercignani, C.: The Boltzmann Equation and Its Applications. Springer, New York (1988)

6. Cieplak, M., Koplik, J., Banavar, J.R.: Molecular dynamics of flows in the Knudsen regime. Physica A 287, 153-160 (2000)

7. Cieplak, M., Koplik, J., Banavar, J.R.: Boundary conditions at a fluid-solid interface. Phys. Rev. Lett. 86, 803-806 (2001)

8. Cercignani, C., Lampis, M.: Kinetic model for gas-surface interaction. Transp. Theory Stat. Phys. 1, 101-114 (1971)

9. Cercignani, C., Lampis, M.: A new scattering kernel for gas-surface interaction. AIAA J. 35(5), 10001011 (1997)

10. Cercignani, C., Lampis, M., Lentati, A.: A new scattering kernel in kinetic theory of gases. Transp. Theory Stat. Phys. 24, 1319-1336 (1995)

11. Darrozes, J.S.: Approximate solutions of the Boltzmann equation for flows past bodies of moderate curvature. In: Trilling, L., Wachman, H.Y. (eds.) Rarefied Gas Dynamics, vol. I, pp. 111-120. Academic Press, New York (1969)

12. Dadzie, S.K., Méolans, J.G.: Anisotropic scattering kernel: generalized and modified Maxwell boundary conditions. J. Math. Phys. 45(5), 1804-1819 (2004)

13. Lauga, E., Brenner, M.P., Stone, H.A.: Microfluidics: the no-slip boundary condition. In: Tropea, C., Yarin, A., Foss, J.F. (eds.) Handbook of Experimantal Fluid Dynamics. Springer, New York (2006) Chap. 15

14. Priezjev, N.V., Troian, S.M.: Influence of periodic wall roughness on the slip behavior at liquid/solid interfaces: molecular-scale simulations versus continuum predictions. J. Fluid. Mech. 554, 25-46 (2006)

15. Qian, T., Wang, X.-P.: Driven cavity flow: from molecular dynamics to continuum hydrodynamics. Multiscale Model. Simul. 3(4), 749-763 (2005)

16. Sone, Y., Aoki, K.: Steady gas flows past bodies at small Knudsen numbers-Boltzmann and hydrodynamic systems. Transp. Theory Stat. Phys. 16, 189-199 (1987)

17. Sharipov, F.: Application of the Cercignani-Lampis scattering kernel to calculation of rarefied gas flow, I: plane flow between two parallel plates. Eur. J. Mech. B/Fluids 21, 113-123 (2002)

18. Sharipov, F.: Application of the Cercignani-Lampis scattering kernel to calculation of rarefied gas flow, II: slip and jump coefficients two parallel plates. Eur. J. Mech. B/Fluids 22, 133-143 (2003)

19. Sharipov, F.: Application of the Cercignani-Lampis scattering kernel to calculation of rarefied gas flow, III: poiseuille flow and thermal creep through a long tube. Eur. J. Mech. B/Fluids 22, 145-154 (2003)

20. Sone, Y.: Asymptotic theory of flow of rarefied gas over a smooth boundary I. In: Trilling, L., Wachman, H.Y. (eds.) Rarefied Gas Dynamics, vol. I, pp. 243-253. Academic Press, New York (1969)

21. Sone, Y.: Asymptotic theory of a steady flow of a rarefied gas past bodies for small Knudsen number. In: Gatignol, R., Soubbaramayer, O. (eds.) Advances in Kinetic Theory and Continuum Mechanics, pp. 1931. Springer, Berlin (1991)

22. Sone, Y.: Kinetic Theory and Fluid Dynamics. Birkhäuser, Boston (2002) 